\title{
PII: S0093-6413(01)00143-4
}

\section{Analysis of a Rubber Cone Tensioned by a Concentrated Force}

\author{
Y C Gao* S H Chen** \\ * Northern Jiaotong University, 100044, Beıjing, Chına \\ ** LNM, Institute of Mechanıcs, Chınese Academy of Sciences, 100080, Beıjıng, Chına
}

(Recelved 10 June 2000, accepted for print 11 December 2000)

Abstract

Using the constitutive equation of a rubber-like materials given by Gao (1997), this paper investigates the problem of a cone under tension of a concentrated force at its apex Under consideration is the axialsymmetry case and the large strain is taken into account The stress strain fields near the apex are obtained by both asymptotic analysis and finite element calculation The two results are consistent well When the cone angle is $180^{\circ}$, the solution becomes that of non-linear Boussinesq's problem for tension case Key words large strain, rubber cone, asymptotic analysis, finite element

\section{Introduction}

A typical problem in nonlinear theory of elasticity is the determination of stress and strain in a rubber cone acted by a concentrated force Gao and Liu (1995) gave a solution to this problem for tension case based on the elastic law proposed by Gao (1990) When the cone angle is $180^{\circ}$, it becomes a rubber half space, the problem can be called nonlınear Boussinesq's problem, which was analyzed for tension case by Simmonds and Warne (1994) based on the elastic law used in Knowles and Sternberg (1973) As for the compression case of the problem, there are still no result published

There are two main obstacles in nonlinear elastic theory, one is the geometry description of deformation, another is the constitutive equation that must be as simple as possible but keep reasonable when strain is large Gao (1997) proposed another elastic law that reflected the response of materials to tension and compression, based on which, the problem of a wedge under compression was solved by Gao (1998)

In the present paper the problem of a cone under tension of a concentrated force is analyzed based on the elastic law of Gao (1997) It is found that the cone apex is in uniaxial tension state, this is similar to that solution obtained by Gao and Liu (1995), however some different material behaviors governed by Gao (1990) and Gao (1997) are revealed in this typical problem

\section{Basıc Formulae}

A three-dimensional domain of rubber material is considered Let $\boldsymbol{P}$ and $\boldsymbol{Q}$ denote the position vectors of a material point before and after deformation respectively, $x^{l}(l=1,2,3)$ is the Lagrangian coordinate Two sets of local triads are defined as,

$$
\boldsymbol{P}_{l}=\partial \boldsymbol{P} / \partial x^{l}, \quad \boldsymbol{Q}_{\boldsymbol{l}}=\partial \boldsymbol{Q} / \partial \boldsymbol{x}^{l}
$$

The displacement gradient tensor is,

$$
\boldsymbol{F}=\boldsymbol{Q}_{l} \otimes \boldsymbol{P}^{l}
$$

where $P^{t}$ is the conjugate of $P_{l}, \otimes$ the dyadic symbol The summation rule is

implied The right and left Cauchy-Green strain tensors are

$$
D=F^{T} F, \quad d=F F^{T}
$$

in which the superscript $T$ denotes transposition $D$ and $d$ possess the same invariants such as

$$
I_{1}=D E=d \quad E, \quad I_{-1}=D^{-1} E=d^{-1} E
$$

where $\boldsymbol{E}$ denotes unit tensor, denotes dual product Besides, a common used invariant is the volume inflation ratio $J$,

$$
J=V_{Q} / V_{P}
$$

where $\quad V_{*}=\left({ }^{*} 1,{ }_{2},{ }^{*}\right)$

\footnotetext{
*Emarlycgao@center njtu edu cn
} 
$\left({ }^{*}, *_{2}, *_{3}\right)$ denotes the mixed product of $*_{1},{ }^{*},{ }^{*}{ }_{3}$

Gao (1997) proposed a strain energy per unit undeformed volume,

$$
U=a\left(I_{1}^{n}+I_{-1}^{n}\right)
$$

where $a, n$ are material constants The Kurchhoff stress is

$$
\sigma=2 \frac{\partial U}{\partial D}=2 n a\left(I_{1}^{n-1} E-I_{-1}^{n-1} D^{-2}\right)
$$

Then the Cauchy stress is

$$
\tau=J^{-1} \boldsymbol{F} \quad \sigma F^{T}=2 n J^{-1}\left(I_{1}^{n-1} d-I_{-1}^{n-1} d^{-1}\right)
$$

The equilibrium equation can be written as

$$
\frac{\partial}{\partial x^{\prime}}\left(V_{Q} \tau Q^{\prime}\right)=0
$$

\section{Mapping Functions and Strain}

A cone before and after deformation is shown in Fig 1 (a), (b) respectively Two Lagrangian coordinates are taken such that $(R, \Theta, \Phi)$ is spherical coordinate before deformation while $(r, \theta, \varphi)$ is spherical coordinate after deformation We presume that the deformation near the apex can be described by the following mapping functions,

$$
\left\{\begin{array}{l}
R=r^{1+\beta} f(\xi) \\
\Theta=g(\xi), \quad \xi=\theta^{-\alpha}, \quad 0 \leq \xi \leq \xi_{0} \\
\Phi=\varphi
\end{array}\right.
$$

Where $\alpha$ and $\beta$ are positive constants to be determined, $f$ and $g$ are unknown functions $\xi_{0}$ is the value of $\xi$ on the cone surface Let

$$
\begin{array}{lll}
\boldsymbol{P}_{R}=\frac{\partial \boldsymbol{P}}{\partial R}=e_{R}, & \boldsymbol{P}_{\Theta}=\frac{\partial \boldsymbol{P}}{\partial \Theta}=R \boldsymbol{e}_{\Theta}, & \boldsymbol{P}_{\Phi}=\frac{\partial \boldsymbol{P}}{\partial \Phi}=R \sin \theta e_{\Phi} \\
\boldsymbol{Q}_{r}=\frac{\partial \boldsymbol{Q}}{\partial}=e_{r}, & \boldsymbol{Q}_{\theta}=\frac{\partial \boldsymbol{Q}}{\partial \theta}=r \boldsymbol{e}_{\theta}, & \boldsymbol{Q}_{\varphi}=\frac{\partial \boldsymbol{Q}}{\partial \varphi}=r \sin \theta e_{\varphi}
\end{array}
$$
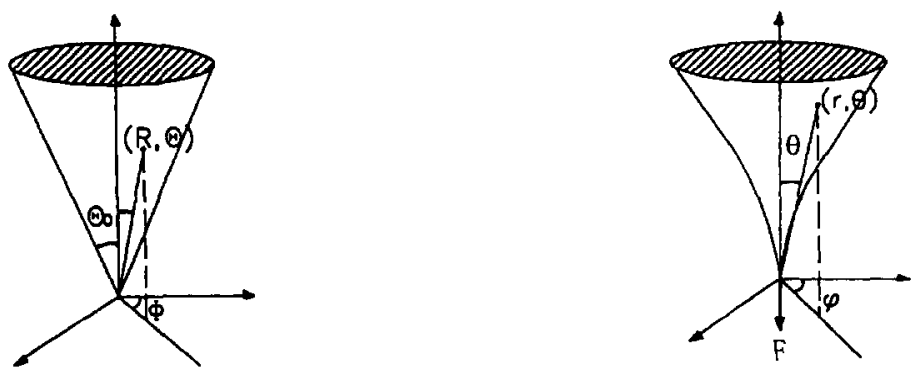

Fig 1 Coordinates
(a) Spherical coordinate before deformation
(b) Spherical coordinate after deformation

evidently, $e_{1}(l=R, \Theta, \Phi, r, \theta, \varphi)$ is unit vector From (11) and (12) it follows

$$
\left\{\begin{array}{l}
\boldsymbol{P}_{r}=\frac{\partial \boldsymbol{P}}{\partial}=r^{\beta}\left\{\left[(1+\beta) f-\alpha \xi f^{\prime}\right] e_{R}-\alpha \xi f g^{\prime} e_{\Theta}\right\} \\
\boldsymbol{P}_{\theta}=\frac{\partial \boldsymbol{P}}{\partial \theta}=r^{1+\beta-\alpha}\left(f^{\prime} \boldsymbol{e}_{R}+f g^{\prime} \boldsymbol{e}_{\Theta}\right) \\
\boldsymbol{P}_{\varphi}=\frac{\partial \boldsymbol{P}}{\partial \varphi}=r^{1+\beta} f \sin g \boldsymbol{e}_{\Phi}
\end{array}\right.
$$


Eq (14) can be inverted to obtain

$$
\begin{aligned}
& \left\{\begin{array}{l}
\boldsymbol{P}^{r}=r^{-\beta} v^{-1}\left(f g^{\prime} e_{R}-f^{\prime} e_{\Theta}\right) \\
\boldsymbol{P}^{\theta}=r^{\alpha-\beta-1} v^{-1}\left\{\alpha \xi f g^{\prime} e_{R}+\left[(1+\beta) f-\alpha \xi f^{\prime}\right] e_{\Theta}\right\} \\
\boldsymbol{P}^{\varphi}=r^{-1-\beta}(f \sin g)^{-1} e_{\Phi}
\end{array}\right. \\
& v=(1+\beta) f^{2} g^{\prime}
\end{aligned}
$$

Using (13)-(15), (2) and (3) we obtain

$$
\begin{aligned}
d= & r^{-2 \beta} v^{-2}\left\{u e_{r} \otimes e_{r}+r^{\alpha}\left[\alpha \xi u-(1+\beta) f f^{\prime}\right]\left(e_{r} \otimes e_{\theta}+e_{\theta} \otimes e_{r}\right)\right. \\
& \left.+r^{2 \alpha}\left[\alpha^{2} \xi^{2} u+(1+\beta)^{2} f^{2}-2(1+\beta) \alpha \xi f f^{\prime}\right] e_{\theta} \otimes e_{\theta}\right\} \\
& +r^{2 \alpha-2 \beta} z^{-2} e_{\varphi} \otimes e_{\varphi} \\
d^{-1} & =r^{2 \beta-2 \alpha}\left\{u e_{\theta} \otimes e_{\theta}-r^{\alpha}\left[\alpha \xi u-(1+\beta) f f^{\prime}\right]\left(e_{r} \otimes e_{\theta}+e_{\theta} \otimes e_{r}\right)\right. \\
& \left.+r^{2 \alpha}\left[\alpha^{2} \xi^{2} u+(1+\beta)^{2} f^{2}-2(1+\beta) \alpha \xi f f^{\prime}\right] e_{r} \otimes e_{r}\right\} \\
& +r^{2 \beta-2 \alpha} z^{2} e_{\varphi} \otimes e_{\varphi}
\end{aligned}
$$

in which

$$
u=f^{\prime 2}+f^{2} g^{\prime 2}, \quad z=f \xi^{-1} \sin g
$$

From(4) - (6), (17), (18), (13) and (14) we have

$$
I_{1}=r^{-2 \beta} v^{-2} u, \quad I_{-1}=r^{2 \beta-2 \alpha}\left(u+z^{2}\right), \quad J=r^{2 \alpha-3 \beta} v^{-1} z^{-1}
$$

\section{Coordinate Transformation}

In order to simplify the expression of $d$ and $d^{-1}$ we introduce a new coordinate system $(\eta, \xi, \varphi)$ in the vicinity of $\theta=0$, on the cross section $\varphi=$ const, the coordinate lines are shown in Fig 2 Then

$$
\eta=r\left(1+\frac{\alpha}{2} \theta^{2}+\frac{\alpha^{2}}{8} \theta^{4}+\frac{\alpha^{3}}{48} \theta^{6}+\right)
$$

The inverse expression of (21) and (11) can be written as

$$
\left\{\begin{array}{l}
r=\eta\left[1-\frac{\alpha}{2}\left(\xi \eta^{\alpha}\right)^{2}\right] \\
\theta=\xi \eta^{\alpha}
\end{array}\right.
$$

According to (22) and neglecting the high order terms, the base vectors in $(\eta, \xi, \varphi)$ system become

$$
\left\{\begin{array}{l}
\boldsymbol{Q}_{\eta}=\frac{\partial \boldsymbol{Q}}{\partial \eta}=\boldsymbol{e}_{r}+\alpha \xi \eta^{\alpha} \boldsymbol{e}_{\theta}, \quad \boldsymbol{Q}_{\xi}=\frac{\partial \boldsymbol{Q}}{\partial \xi}=\eta^{1+\alpha}\left(\boldsymbol{e}_{\theta}-\alpha \xi \eta^{\alpha} e_{r}\right) \\
\boldsymbol{Q}_{\varphi}=\frac{\partial \boldsymbol{Q}}{\partial \varphi}=\xi \eta^{1+\alpha} e_{\varphi}
\end{array}\right.
$$

then

$$
\left\{\begin{array}{l}
Q^{\eta}=e_{r}+\alpha \xi \eta^{\alpha} e_{\theta}, \\
Q^{\varphi}=\xi^{-1} \eta^{-1-\alpha} e_{\varphi}
\end{array} \quad Q^{\xi}=\eta^{-1-\alpha}\left(e_{\theta}-\alpha \xi \eta^{\alpha} e_{r}\right)\right.
$$

The unit vectors along $\eta$ and $\xi$ lines are

the inverse of (25) is

$$
e_{\eta}=e_{r}+\alpha \theta e_{\theta}, \quad e_{\xi}=-\alpha \theta e_{r}+e_{\theta}
$$

$$
e_{r}=e_{\eta}-\alpha \xi \eta^{\alpha} e_{\xi}, \quad e_{\theta}=e_{\xi}+\alpha \xi \eta^{\alpha} e_{\eta}
$$

Substituting (22) and (26) into (17), (18) and (20), it is obtained

$$
\begin{aligned}
& d=\eta^{-2 \beta}\left[u e_{\eta} \otimes e_{\eta}-\eta^{\alpha}(1+\beta) f f^{\prime}\left(e_{\eta} \otimes e_{\xi}+e_{\xi} \otimes e_{\eta}\right)\right. \\
& \left.+\eta^{2 \alpha}(1+\beta)^{2} f^{2} e_{\xi} \otimes e_{\xi}\right]+\eta^{2 \alpha-2 \beta} z^{-2} e_{\varphi} \otimes e_{\varphi} \\
& d^{-1}=\eta^{2 \alpha-2 \beta}\left[u e_{\xi} \otimes e_{\xi}+\eta^{\alpha}(1+\beta) f f^{\prime}\left(e_{\eta} \otimes e_{\xi}+e_{\xi} \otimes e_{\eta}\right)\right. \\
& \left.+\eta^{2 \alpha}(1+\beta)^{2} f^{2} e_{\eta} \otimes e_{\eta}\right]+\eta^{2 \beta-2 \alpha} z^{2} e_{\varphi} \otimes e_{\varphi}
\end{aligned}
$$



Fig $2(\xi, \eta)$ coordinate

Substituting (27), (28) into (9), if we require that both $d$ and $d^{-1}$ make contribution to the coefficient of 
$e_{\xi} \otimes e_{\xi}$, then the following condition is obtained,

$$
\alpha=2 n \beta /(n+1)
$$

Further, the resultant force of $\tau$ must balance the external load, so we have

$$
r^{2(1+\alpha)} \tau^{r r} \sim 1
$$

therefore

$$
\beta=\frac{2}{2 n-3}, \quad \alpha=\frac{4 n}{(2 n-3)(n+1)}
$$

finally $\tau$ can be written as

$$
\begin{aligned}
\tau & =2 n a \eta^{-\lambda}\left[T e_{\eta} \otimes e_{\eta}+\eta^{\alpha} S\left(e_{\eta} \otimes e_{\xi}+e_{\xi} \otimes e_{\eta}\right)\right. \\
& \left.+\eta^{2 \alpha}\left(w e_{\xi} \otimes e_{\xi}+Y e_{\varphi} \otimes e_{\varphi}\right)\right]
\end{aligned}
$$

in which

$$
\begin{aligned}
& \lambda=2(1+\alpha) \\
& \left\{\begin{array}{l}
T=v^{1-2 n} z u^{n} \\
S=-v^{1-2 n} z u^{n-1}(1+\beta) f f^{\prime} \\
w=v z\left[v^{-2 n} u^{n-1}(1+\beta)^{2} f^{2}-\left(u+z^{2}\right)^{n-1} u\right] \\
Y=v z\left[v^{2-2 n} u^{n-1} z^{-2}-\left(u+z^{2}\right)^{n-1} z^{2}\right]
\end{array}\right.
\end{aligned}
$$

\section{Equilibrium Equation and Solution}

In order to use equation (10), the following relatıons for dominant terms are needed,

$$
\left\{\begin{array}{l}
\frac{\partial e_{\eta}}{\partial \eta}=\alpha(1+\alpha) \xi \eta^{\alpha-1} e_{\xi}, \frac{\partial e_{\eta}}{\partial \xi}=(1+\alpha) \eta^{\alpha} e_{\xi}, \frac{\partial e_{\eta}}{\partial \varphi}=(1+\alpha) \xi \eta^{\alpha} e_{\varphi} \\
\frac{\partial e_{\xi}}{\partial \eta}=-\alpha(1+\alpha) \xi \eta^{\alpha-1} e_{\eta}, \frac{\partial e_{\xi}}{\partial \xi}=-(1+\alpha) \eta^{\alpha} e_{\eta}, \frac{\partial e_{\xi}}{\partial \varphi}=e_{\varphi} \\
\frac{\partial e_{\varphi}}{\partial \eta}=0, \quad \frac{\partial e_{\varphi}}{\partial \xi}=0, \quad \frac{\partial e_{\varphi}}{\partial \varphi}=-e_{\xi}
\end{array}\right.
$$

Besides, Eqs (23) and (6) give

$$
V_{Q}=\eta^{2+2 \alpha} \xi
$$

Substituting (32)-(36) and (24) into (10) and neglecting the high order terms, it is obtained,

$$
\left\{\begin{array}{l}
\frac{d S}{d \xi}+\frac{S}{\xi}=0 \\
\frac{d w}{d \xi}+\frac{1}{\xi}(w-Y)+\alpha(1+\alpha) \xi T+(1+2 \alpha) S=0
\end{array}\right.
$$

Let $\xi_{0}$ denote the value of $\xi$ on the cone surface, $\Theta_{0}$ the half angle of the cone apex, then we have $g\left(\xi_{0}\right)=\Theta_{0}$

At the surface of the cone, traction free condition must be satısfied, so that at $\xi=\xi_{0}$ it is required,

$$
\left.S\right|_{\xi_{0}}=0,\left.\quad w\right|_{\xi_{0}}=0
$$

From the first of (37) and the first of (39), it is concluded that

$$
S \equiv 0
$$

(40) results in

$$
f=f_{0}=\text { const }
$$

Then

$$
u=f_{0}^{2} g^{\prime 2}, \quad v=(1+\beta) f_{0}^{2} g, \quad z=f_{0} \xi^{-1} \sin g
$$

Substitutıng (34) and (42) into the second of (37), it follows 
in which

$$
\begin{aligned}
& g^{n} \sin g\left\{1-(1+\beta)^{2 n-2} f_{0}^{4 n} g^{\prime 4} B^{n}{ }^{2}\left[(2 n+1) g^{\prime 2}+3 A^{2}\right]\right\} \\
& -g^{\prime 2}\left[\cos g-\frac{g^{\prime}}{A}+\frac{\alpha(1+\alpha)}{(1+\beta)^{2}} \xi g^{\prime} \sin g\right]-(1+\beta)^{2 n-2} f_{0}^{4 n} g^{\prime 3} B^{n-2}\left[A^{5}\right. \\
& \left.-g^{\prime 5} \cos g+(2 n-1) g^{\prime 2} A^{2}\left(A-g^{\prime} \cos g\right)\right]=0
\end{aligned}
$$

At $\xi=0$ we have

$$
A=\xi^{-1} \sin g, \quad B=g^{\prime 2}+A^{2}
$$

$$
g(0)-0
$$

(38) and the second of (39) can be combined to give,

$$
g^{\prime 4} B^{n-1}=(1+\beta)^{2-2 n} f_{0}^{-4 n} \quad \text { when } g=\Theta_{0}
$$

(45) and (46) are the two boundary conditions for Eq (43) so that it can be solved numerically Eqs (43)(46) show that if $f_{0}-g(\xi)$ is a set of solution, then $k f_{0}-g\left(\xi k^{-\alpha / \beta}\right)$ is also a set of solution Therefore it is sufficient to give the numerical calculation only for $f_{0}=1$ For $n=2, f_{0}=1, \Theta_{0}=\pi / 6, \pi / 4, \pi / 2$, the curves of $g(\xi)$ are shown in Fig 3 It can be seen that the curves of $g(\xi)$ are nearly straight lines

Now, we should determine the relation of $f_{0}$ and the load $F$ at the apex Let $n$ denote the normal of the cross section $r-$ const then by integral we have

$$
\begin{aligned}
F & =2 \pi r^{2} \int \tau n \sin \theta d \theta \\
& =4 \pi n a \int_{0}^{\xi_{0}} T \quad \xi d \xi=4 \pi n a(1+\beta)^{1-2 n} f_{0}^{3-2 n}\left(1-\cos \Theta_{0}\right)
\end{aligned}
$$

or

$$
f_{0}=\left[\frac{F}{4 \pi n a\left(1-\cos \Theta_{0}\right)}\right]^{-\frac{\beta}{2}}(1+\beta)^{-1-\beta}
$$

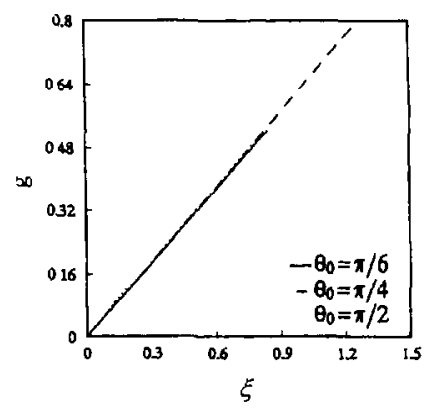

Fig 3 Curves of $\xi \sim g$

\section{Finite Element Calculation}

The incremental theory of total Lagrangian approach is adopted in the finite element calculation Under consideration is the axial symmetry case The mesh division in the vertical cross section of the cone is shown in Fig 4 In the $R$ direction the cone is divided into 50 layers and the size ratio of neighboring elements is 12 In $\Theta$ direction it is equally divided into 8 layers The generatrix length is taken to be 15 The material parameters are taken as $n=25, a=150000, F=30, \Theta_{0}=\pi / 6$ The calculated curve of $R(r)$ and the theoretical curve from (11) for $\Theta=0$ are plotted in Fig 5 The curves of $r \sim \tau^{r r}$ for $\Theta=0$ are shown in Fig 6 The shape of the vertical cross section near the apex after deformation is shown in Fig 7 It is shown that when $r$ is small enough, the numerical results are consistent with the asymptotic analysis For other parameters, for examples $n=20, a=150000, F=30, \Theta_{0}=\pi / 6$ or $\Theta_{0}=\pi / 4$, the curves are very similar to Fig 5 and Fig 6 so they are not plotted

\section{Conclusion and Discussion}

- The asymptotic analysis and finite element calculation revealed that when a cone is tensioned by a concentrated force, the stress state near the apex is in uniaxial tension The stress component $\tau^{r r}$ possesses a constant angular distribution This is similar to that obtained by Gao and Liu (1995)

- With the solution of Eq (43), the minor components of stress ( $\tau^{\xi \xi}$ and $\tau^{\varphi \varphi}$ ) can be determined However for the same problem but based on another elastic law, Gao (1990), the minor stress component $\tau^{\theta \theta}, \tau^{\varphi \varphi}$ cannot be determined near the apex Therefore, we conclude that the new elastic law, Gao (1997), can reflect the material behavior more exactly than the old elastic law, Gao (1990)

- Eq (31) shows that $n>15$ is the precondition for the materials to endure a concentrated force This is consistent with the analysis given by Gao and Gao (1999) 

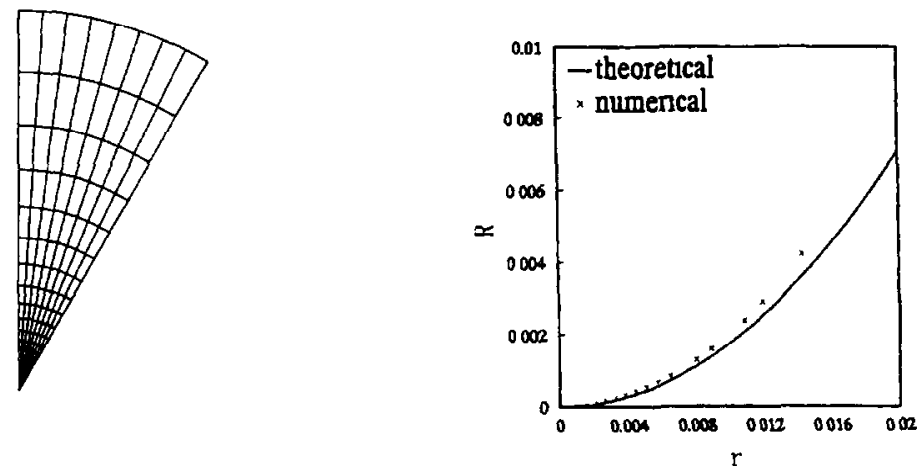

Fig 4 Mesh division of a vertıcal cross section

Fig 5 Curves of $r \sim R$
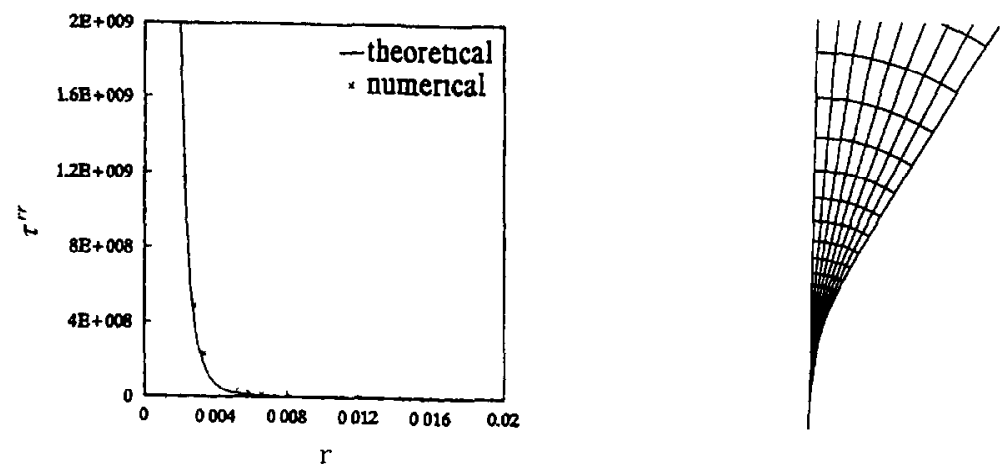

Fig 6 Curves of $r \sim \tau^{r r} \xi$

Fig 7 Shape of the vertical cross section after deformation

\section{Acknowledgment}

This work is supported by the National Science Foundation of China (19572001 and 19772001)

\section{References}

Gao, Y C (1990), Elastostatıc crack tıp behavıor for a rubber-like materıal, Theor Appl Fract Mech Vol 14, PP 219-231

Gao, Y C and Liu, B (1995), A rubber cone under the tension of a concentrated force, Int J Solids Struct Vol 32, PP 1485-1493

Gao, Y C (1997), Large deformation field near a crack tıp in rubber-like material, Theor Appl Fract Mech Vol 26, PP 155-162

Gao, Y C (1998), Large strain analysıs of a rubber wedge compressed by a line load at its tip, Int J Eng Sc1 Vol 36, PP 831-842

Gao, Y C and Gao, T (1999), Mechanıcal behavior of two kınds of rubber materials, Int J Solids Struct, Vol 36, PP 5545-5558

Knowles, J K and Sternberg, E (1973), An asymptotic finite deformation analysis of the elastic field near the tip of a crack, J Elasticity Vol 3, PP 67-107

Simmonds, J G and Warne, P G (1994), Notes on the nonlinearly elastic Boussinesq's problem, J Elastıcity, Vol 34, PP 69-82 